\title{
Hedgehog signalling does not stimulate cartilage catabolism and is inhibited by Interleukin-1 $\beta$
}

Clare L. Thompson ${ }^{1}$, Riana Patel ${ }^{1}$, Terri-Ann N. Kelly ${ }^{1,3^{*}}$ (D, Angus K. T. Wann ${ }^{1,4}$, Clark T. Hung ${ }^{3}$, J. Paul Chapple ${ }^{2}$ and Martin M. Knight ${ }^{1}$

\begin{abstract}
Background: In osteoarthritis, chondrocytes adopt an abnormal hypertrophic morphology and upregulate the expression of the extracellular matrix-degrading enzymes, MMP-13 and ADAMTS-5. The activation of the hedgehog signalling pathway has been established in osteoarthritis and is thought to influence both of these processes. However, the role of this pathway in the initiation and progression of osteoarthritis is unclear as previous studies have been unable to isolate the effects of hedgehog pathway activation from other pathological processes. In this study we test the hypothesis that hedgehog pathway activation causes cartilage degradation in healthy cartilage and in an in vitro model of inflammatory arthritis.
\end{abstract}

Methods: Isolated articular chondrocytes from the bovine metacarpal-phalangeal joint were stimulated for up to 24 hours with the agonist, recombinant Indian hedgehog (r-Ihh). ADAMTS-5 and MMP-13 gene expression was quantified by real-time PCR. In addition, healthy bovine cartilage explants were treated with r-lhh or the hedgehog antagonist, cyclopamine, and sGAG release into the media was measured over 72 hours. Studies were repeated using chondrocytes and cartilage explants from human knee joint. Finally, studies were conducted to determine the effect of hedgehog pathway activation on matrix catabolism in the presence of the pro-inflammatory cytokine, IL-1ß.

Results: Addition of r-Ihh activated hedgehog signalling, confirmed by upregulation of Gli1 and Ptch1 expression, but did not increase ADAMTS-5 or MMP-13 expression in bovine or human chondrocytes. Furthermore, r-lhh did not induce sGAG release in healthy bovine or human cartilage explants. IL-1 $\beta$ treatment induced sGAG release, but this response was not altered by the stimulation or inhibition of hedgehog signalling. Hedgehog pathway activation was downregulated by IL-1ß. Conversely, r-lhh weakly suppressed IL-1ß-induced ADAMTS-5 expression.

Conclusion: Our results show for the first time that Indian hedgehog does not cause extracellular matrix degradation in healthy ex vivo cartilage or in the presence of IL-1 $\beta$ and that IL-1 $\beta$ downregulates Indian hedgehog induced signalling. Thus, we suggest reported hedgehog induced matrix catabolism in osteoarthritis must be due to its interaction with pathological factors other than IL-1 $\beta$. Hence, hedgehog signalling and its downstream effects are highly context-dependent.

Keywords: Osteoarthritis, Articular cartilage, Hedgehog signal transduction, Interleukin 1 $\beta$, Cyclopamine, Indian hedgehog

\footnotetext{
*Correspondence: t.a.kelly@qmul.ac.uk

Clare L. Thompson and Riana Patel joint first authors.

${ }^{1}$ Institute of Bioengineering and School of Engineering and Material Sciences,

Queen Mary University of London, Mile End Road, London E1 4NS, UK

${ }^{3}$ Department of Biomedical Engineering, Columbia University, New York, NY, USA

Full list of author information is available at the end of the article
} 


\section{Background}

Osteoarthritis (OA), characterised by the progressive degeneration of load-bearing diarthrodial joints, such as the knees and hips, is one of the leading causes of disability worldwide. In OA, the metabolic balance between extracellular matrix production and degradation is disrupted, leading to increased catabolic breakdown of the articular cartilage [1]. The mechanisms underlying OA are not fully understood, however, previous research indicates that disease progression is influenced by a combination of factors including injury, metabolism, age, and genetics [2]. Furthermore, strong evidence points to a significant inflammatory component to $\mathrm{OA}$, involving cytokine cross-talk between chondrocytes, the synovium lining the joint capsule and the underlying bone [3].

The hedgehog signalling pathway has also been implicated in the pathogenesis of OA [4-6]. In articular cartilage, hedgehog pathway activation requires the binding of Indian hedgehog (Ihh) to its transmembrane receptor, Patched-1 (Ptch1). Ptch1 causes disinhibition of the transmembrane protein Smoothened (Smo), enabling its enrichment and activation in the primary cilium, a microtubule-based organelle found in most cells, including articular chondrocytes [7-9]. Smo indirectly activates the downstream Gli transcription factors, which in turn translocate to the nucleus to induce the expression of hedgehog target genes, such as Ptch1 and Gli1 [7, 8, 10, 11]. Hedgehog signalling regulates chondrocyte differentiation and proliferation during embryonic skeletal development and in the terminal growth plate $[12,13]$. In OA, chondrocytes exhibit a phenotypic shift to a more terminal growth-plate-like state, adopting a similar hypertrophic morphology and upregulating the expression of hypertrophic markers, including type $\mathrm{X}$ collagen, matrix metalloproteinase (MMP)-13 and a disintegrin and metalloproteinase with thrombospondin motifs (ADAMTS)-5 [14]. Hedgehog pathway activation is thought to contribute to this process. Indeed, Lin et al. report that hedgehog pathway activation is increased in both human and mouse OA cartilage, with higher levels of hedgehog signalling correlating with increased disease severity [5]. Moreover, hedgehog blockade reduces the expression of OA-related genes, including ADAMTS5 and $M M P 13$, in mice with surgically induced OA and in human OA cartilage samples $[5,6]$. Further evidence for the role of hedgehog pathway activation in OA stems from the increased expression of Indian hedgehog (Ihh) in cartilage and synovial fluid in human OA, with a 5.2-fold and a 1.71-fold increase, respectively, compared to their normal controls $[4,15]$.

Hedgehog pathway activation stimulates specific Gli2mediated expression of runt-related transcription factor 2 (Runx2) [16]. Runx2 upregulates several matrix-degrading enzymes, including MMPs-1, 3, and 13 and ADAMTS-4 and ADAMTS-5, increases production of nitric oxide
(NO) and prostaglandin $\mathrm{E}_{2}\left(\mathrm{PGE}_{2}\right)$ and upregulates the expression of type $\mathrm{X}$ collagen. The Ihh-Runx2 pathway thus represents a plausible mechanism by which the hedgehog pathway influences OA development as Runx2 functions upstream of many proteins identified in cartilage lesions in early OA $[5,17,18]$.

While hedgehog pathway activation has been demonstrated to increase the likelihood of developing OA, the nature of these studies prevent analysis of hedgehog pathway activation in isolation from other processes that occur within the complex environment of the joint. In the current study we test if hedgehog pathway activation alone can stimulate cartilage degradation in healthy human and bovine articular cartilage samples. We report that pathway activation in response to Indian hedgehog (Ihh) does not influence the expression of ADAMTS-5 or $M M P-13$, nor induce cartilage degradation, as measured by sulphated glycosaminoglycan (sGAG) release. The response to hedgehog pathway activation was also examined in an in vitro bovine model of inflammatory arthritis. We report that neither stimulation nor inhibition of the hedgehog pathway influenced cartilage degradation in the presence of IL-1 $\beta$. Thus, we conclude that the catabolic effects of hedgehog signalling in $\mathrm{OA}$ must arise due to interaction with other physiological or pathological signalling pathways within the joint.

\section{Methods}

\section{Reagents and media preparation}

Chondrogenic media consisted of DMEM supplemented with $86 \mathrm{U} / \mathrm{mL}$ penicillin-streptomycin, $1.72 \mathrm{mM} \mathrm{L}$ glutamine, $17.25 \mathrm{mM}$ HEPES, $0.12 \mathrm{mg} / \mathrm{mL}$ ascorbate, and 10 \% FBS (Sigma-Aldrich, Poole, UK). Recombinant human IL-1 $\beta$ (Peprotech, London, UK) stock solution consisted of $10 \mu \mathrm{g} / \mathrm{mL}$ in PBS containing $0.01 \%$ BSA and $10 \%$ FBS [19]. Recombinant human/mouse Ihh (r-Ihh) stock solution consisted of $200 \mu \mathrm{g} / \mathrm{mL}$ in PBS containing $0.01 \%$ BSA (R\&D Systems, Minneapolis, MN, USA). Cyclopamine stock solution was $10 \mu \mathrm{M}$ in ethanol (EMD Millipore, San Diego, CA, USA). The effective doses and modes of action of the pharmaceuticals used in this study are listed in Table 1. The differences in the cyclopamine doses used for bovine and human samples were based on previous findings [20-24].

\section{Chondrocyte isolation and culture}

Primary bovine articular chondrocytes were isolated from the metacarpophalangeal joints of 1-2-year-old steers by enzymatic digestion as previously described [25]. Human primary articular chondrocytes from the tibial plateau of knee joints from three donors (one female and two male) aged 26-60 years, were purchased from Articular Engineering (Northbrook, IL, USA) and cultured without passage. Primary chondrocytes were 
Table 1 The doses and modes of actions of hedgehog pharmaceuticals

\begin{tabular}{llll}
\hline Drug name & Molecular weight & Mechanism of action & Dose \\
\hline r-Ihh & $20,000 \mathrm{Da}$ & Binds to Ptch1, causing Ptch1 inhibition and Smo activation [5, 41] & $1 \mu \mathrm{g} / \mathrm{mL}$ \\
Cyclopamine & $411.6 \mathrm{Da}$ & Smo antagonist [20-24] & Bovine: $10 \mu \mathrm{M}$ \\
& & & Human: $20 \mu \mathrm{M}$ \\
KAAD-cyclopamine & $698 \mathrm{Da}$ & Smo antagonist $[24,42,43]$ & $1 \mu \mathrm{M}$ \\
SANT-1 & $373.5 \mathrm{Da}$ & Smo antagonist $[20,23,44]$ & $1 \mu \mathrm{M}$ \\
GANT61 & $429.6 \mathrm{Da}$ & Gli antagonist $[39,45,46]$ & $10 \mu \mathrm{M}$ \\
\hline
\end{tabular}

Ptch 1 patched 1, $r$-lhh recombinant Indian hedgehog, Smo smoothened

plated at 80,000 cells per $\mathrm{cm}^{2}$ onto glass coverslips and cultured in chondrogenic media for 5 days (confluence) before treatment with $10 \mathrm{ng} / \mathrm{mL}$ IL- $1 \beta$ or $\mathrm{r}$-Ihh (Table 1 ). After treatment, cultures were processed for real-time PCR as indicated below.

\section{Cartilage explant model}

Full-thickness human articular cartilage explants $(3 \times$ $3 \mathrm{~mm}$ blocks) were harvested from macroscopically healthy regions of the tibial plateau of a male patient undergoing total joint arthroplasty for OA. The human tissue was obtained with informed patient consent and full National Health Service (NHS) ethical approval (East London and The City Research Ethics Committee - Ethics number: 07/Q0605/29). Full-thickness articular cartilage explants (5 mm diameter) were harvested metacarpophalangeal joints from 1-2-year-old steers as previously described [15]. Individual cartilage explants were then placed in 24-well plates and cultured in $1 \mathrm{~mL}$ of their respective experimental media as outlined in Table 1 . The culture media was collected and replaced every 24 hours over the 72 -hour culture period and stored at $-20{ }^{\circ} \mathrm{C}$ for biochemical assay. In the IL-1 $\beta$-treated groups, the media were further supplemented with $2.5 \mathrm{ng} / \mathrm{mL}$ or $10 \mathrm{ng} / \mathrm{mL}$ IL-1 $\beta$ during the first 24 hours in culture.

\section{Explant viability analysis}

Following the 72-hour culture period, a cell viability assay was performed on representative bovine explants from all groups via LIVE/DEAD Cell Viability Assays (Molecular Probes, Paisley, UK) and imaged on a confocal microscope (Leica Microsystems, Wetzlar, Germany). The numbers of viable and dead cells in each field of view were counted using Image (NIH, Bethesda, MD, USA) and used to calculate the percentage cell viability for each group.

\section{Sulphated glycosaminoglycan assay}

Culture media was analysed using the 1,9-dimethylmethylene blue (DMMB) assay to determine the amount of sGAG released due to breakdown of the cartilage extracellular matrix [26]. Each sample was run in triplicate against a chondroitin sulfate standard curve $(0-50 \mu \mathrm{g} / \mathrm{mL})$. As all samples were of similar weights and dimensions, the
sGAG release for each explant was normalized to the mean sGAG release of the untreated explants at the same time point to account for donor variability.

\section{RNA extraction, cDNA synthesis, and real-time PCR}

RNA was extracted from isolated chondrocyte culture and converted to cDNA using the RNeasy and QuantiTect Reverse Transcription Kits (Qiagen, Manchester, UK), evaluated by gel electrophoresis, and quantified using spectrophotometry. Quantitative real-time PCR was performed as previously described [9]. The sequences of the primers used in this experiment are shown in Table 2. Fold-change in gene expression was calculated relative to 18S rRNA controls.

\section{Statistical analysis}

Statistical differences in gene expression, sGAG and cell viability were measured using two-way analysis of variance (ANOVA) with Tukey's post hoc comparison (GraphPad,

Table 2 Primer sequences for the genes measured in this experiment

\begin{tabular}{|c|c|c|}
\hline Gene & Species & Sequence $\left(5^{\prime}-3^{\prime}\right)$ \\
\hline \multirow[t]{2}{*}{ Patched 1} & Bovine & F-ATGTCTCGCACATCAACTGG \\
\hline & & R-TCGTGGTAAAGGAAAGCACC \\
\hline \multirow[t]{2}{*}{ Gli1 } & Bovine & F-ACCCCACCACCAGTCAGTAG \\
\hline & & R-TGTCCGACAGAGGTGAGATG \\
\hline \multirow[t]{2}{*}{ ADAMTS-5 } & Bovine & F- GCCCTGCCCAGCTAACGGTA \\
\hline & & R- CCCCCGGACACACACGGAA \\
\hline \multirow[t]{2}{*}{ MMP-13 } & Bovine & F- CCCTTGATGCCATAACCAGT \\
\hline & & R- GCCCAAAATTITCTGCCTCT \\
\hline \multirow[t]{2}{*}{$18 S$ rRNA } & Bovine & F- GCAATTATTCCCCATGAACG \\
\hline & & R- GGCCTCACTAAACCATCCAA \\
\hline \multirow[t]{2}{*}{ ADAMTS-5 } & Human & F- CCTTGTGGAAAGGGGAGAAT \\
\hline & & R- ACAGTGACGATAGGCAAACT \\
\hline \multirow[t]{2}{*}{ MMP-13 } & Human & F- AGCCACTTTATGCTTCCTGA \\
\hline & & R- TCAAACTGTATGGGTCCGTT \\
\hline \multirow[t]{2}{*}{$18 S$ rRNA } & Human & F- CGGCTACCACATCCAAGGAA \\
\hline & & R- AGCTGGAATTACCGCGGC \\
\hline
\end{tabular}

ADAMTS-5 a disintegrin and MMP with thrombospondin motifs-5, MMP-13 matrix metalloproteinase-13, 18S rRNA $18 \mathrm{~S}$ ribosomal RNA 
La Jolla, CA, USA). The threshold for statistical significance was set at $P<0.05$. All values are displayed as mean \pm standard deviation.

\section{Results}

R-Ihh activates the hedgehog pathway but does not stimulate ADAMTS-5 and MMP-13 expression in bovine articular chondrocytes

Isolated primary bovine chondrocytes were stimulated with r-Ihh for 24 hours to assess the effects of hedgehog pathway activation on catabolic gene expression. Following r-Ihh treatment, Gli1 and Ptch1 expression was increased relative to the unstimulated control by a magnitude of 4.5 -fold $(P \leq 0.001)$ and 3.2-fold $(P \leq 0.01)$ respectively (Fig. 1a). The expression of $M M P-13$ was not significantly altered following $\mathrm{r}$-Ihh treatment, however, a small but significant reduction in ADAMTS-5 expression was observed (0.5-fold, $P \leq 0.01$ ) (Fig. 1b). These results demonstrate that $r$-Ihh successfully activated the hedgehog pathway but did not upregulate catabolic gene expression.

\section{Hedgehog pathway modulation does not alter cartilage degradation in bovine cartilage explants}

The induction of mRNA does not always correlate with catabolic effects, therefore the effect of hedgehog pathway activation on cartilage degradation was directly investigated in bovine cartilage explants. Explants were treated with r-Ihh for 24, 48 and $72 \mathrm{hrs}$ and cartilage degradation monitored by measuring the release of sGAG into the culture media (Fig. 2a). No significant change in sGAG release was observed at any time point relative to the untreated control. Consequently, there was no significant difference in the cumulative sGAG release at the end of the 72-hour culture period. Furthermore, no significant effects on sGAG release were observed when the treatment period was extended (Additional file 1: Figure S1).

The hedgehog pathway antagonist, cyclopamine, was similarly administered to bovine cartilage explants to examine the influence of endogenous basal hedgehog signalling on matrix catabolism. Cyclopamine significantly decreased sGAG release only at the $24-48$ hour time point $(P<0.05)$, at every other time point cyclopamine had no effect on sGAG release and there was no significant difference in cumulative sGAG release at the end of the 72-hour culture period (Fig. $2 b$ ). These results indicate that both hedgehog pathway activation by $r$-Ihh and inhibition by cyclopamine does not influence matrix catabolism in bovine cartilage. Cell viability analysis following treatment showed that the majority of cells are alive after the 72-day treatment period (Additional file 1: Figure S2A), with $\mathrm{r}$-Ihh and cyclopamine treatment yielding similar cell viability as control (Additional file 1: Figure S2b).

\section{Hedgehog pathway activation has no effect on ADAMTS- 5 or MMP-13 expression or matrix catabolism in human cartilage}

The above studies were repeated using human chondrocytes and cartilage explants to examine whether the absence of a catabolic response to r-Ihh was due to the use of bovine tissue. In isolated primary human chondrocytes, r-Ihh stimulation for either 24 or 72 hours did not significantly alter ADAMTS-5 gene expression relative to the untreated control (Fig. 3a and b). A significant 0.6fold reduction in $M M P-13$ expression was observed at 24 hours $(P<0.05$, Fig. 3a), however, by 72 hours this was no longer significantly different to the control group (Fig. 3b). Neither r-Ihh nor cyclopamine significantly altered sGAG release in human cartilage explants (Fig. 3c).
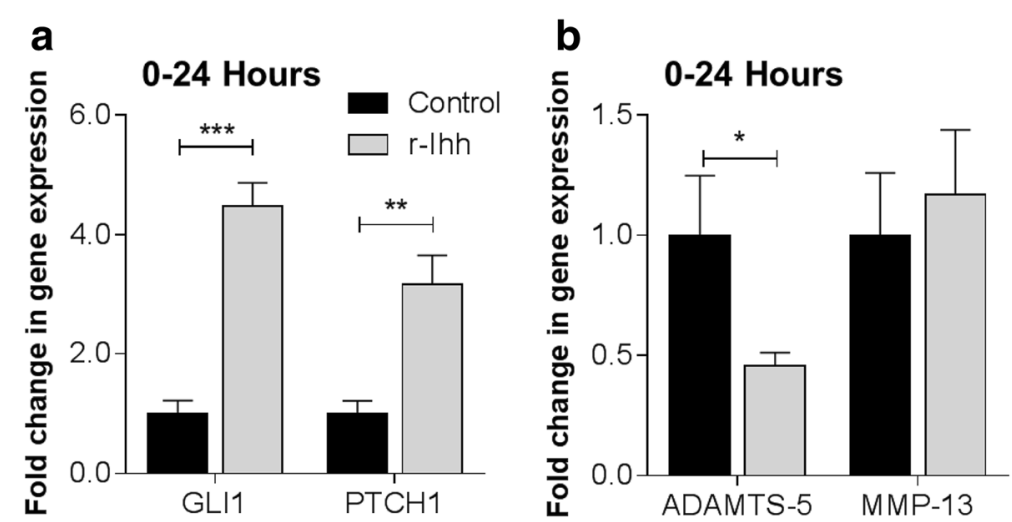

Fig. 1 Hedgehog pathway activation does not increase the expression of a disintegrin and matrix metalloproteinase with thrombospondin motif (ADAMTS-5) and matrix metalloproteinase-13 (MMP-13) in bovine articular chondrocytes. After 24 hours of stimulation with r-lhh, changes in the gene expression of (a) Gli1 and Ptch1 and (b) ADAMTS-5 and MMP-13 were measured. Data are mean fold-change normalised to untreated controls with standard deviation error bars ( $n=9$ from three separate donors). *Statistically significant differences relative to untreated controls, ${ }^{*} P<0.05,{ }^{*} P \leq 0.01$, and ${ }^{* * *} P \leq 0.001$. $r$-lhh recombinant Ihh 

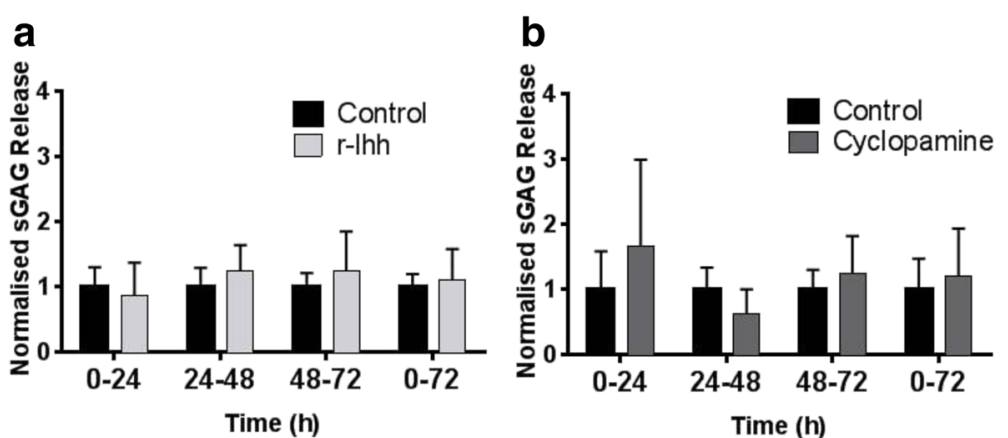

Fig. 2 Hedgehog pathway modulation does not influence cartilage degradation in bovine explants. The sulphated glycosaminoglycans (sGAG) release for explants treated with r-Ihh (a) and cyclopamine (b) shown at 24-hour intervals and cumulatively over the 72-hour culture period as compared to untreated controls. All data are displayed as mean sGAG release, normalised to the untreated controls at the same time point, with standard deviation error bars ( $\mathrm{n}=9$ from three separate donors). $r$-lhh recombinant Ihh

Similar to bovine tissue, these results suggest that hedgehog signalling plays a minimal role in modulating extracellular matrix catabolism in human articular cartilage.

\section{Hedgehog pathway modulation does not affect IL-1 $\beta$ - induced cartilage degradation in bovine cartilage explants}

Next, we sought to examine whether the previously reported degradative effects of hedgehog signalling were due to the presence of inflammatory cytokines in the OA microenvironment. Bovine cartilage explants were treated with $2.5 \mathrm{ng} / \mathrm{mL}$ IL- $1 \beta$ treatment for 24 hours. IL$1 \beta$ treatment resulted in a significant increase in sGAG release over the 72-hour culture period compared to untreated controls $(P<0.01)$ (Fig. 4c). Following the 24 -hour IL-1 $\beta$ treatment, explants displayed a 7.8 -fold increase in sGAG compared to untreated controls $(P<0.05)$ (Fig. 4a). Thereafter, sGAG release decreased at each subsequent time point, with IL-1 $\beta$-treated explants displaying 2.8-fold and 1.3-fold increases relative to controls at 48 and 72 hours, respectively, as the effects of the single dose treatment wore off. To investigate whether IL$1 \beta$ influences the effect of hedgehog pathway activation on cartilage degradation, we evaluated the effects of $r$-Ihh and cyclopamine on sGAG release using this inflammatory model. The release of sGAG over 72 hours was not significantly modulated by the addition of r-Ihh (Fig. 4b) or cyclopamine (Fig. 4c) compared to IL-1 $\beta$ treatment alone. Additional antagonists, KAAD-cyclopamine, SANT1, and GANT61, all showed a similar trend with no significant change in IL-1 $\beta$-induced sGAG release at 72 hours (Additional file 1: Figure S3). Taken together, these results
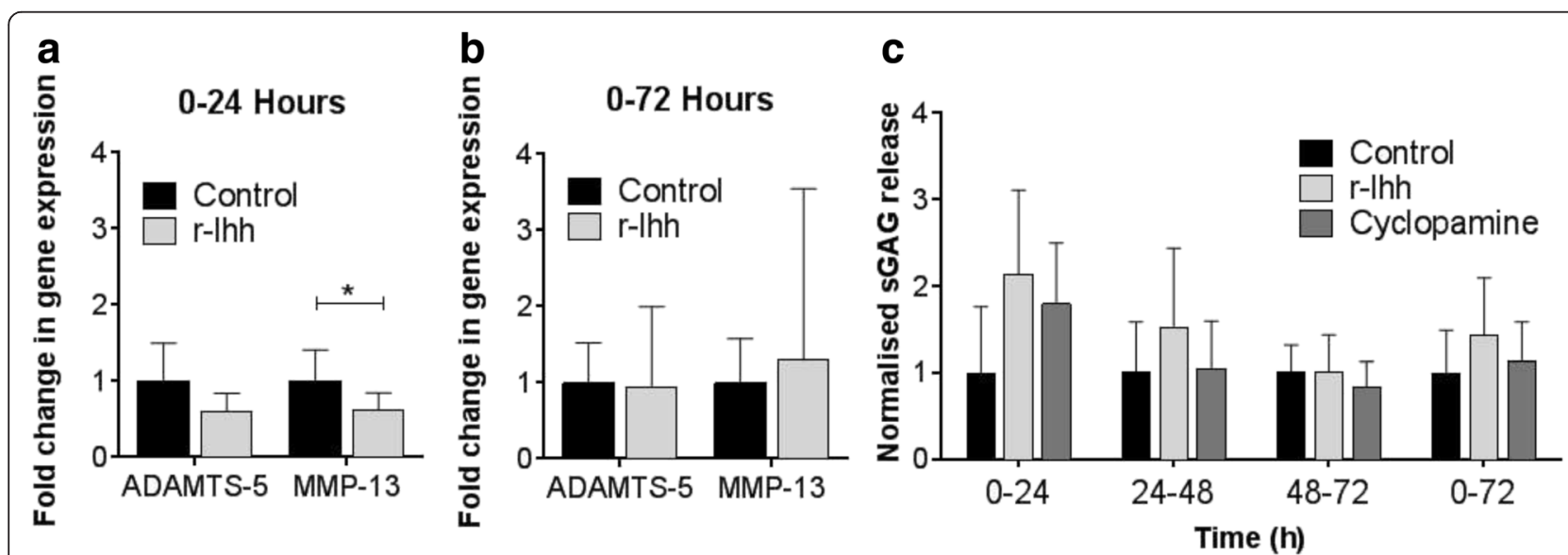

Fig. 3 Hedgehog pathway activation has no effect on a disintegrin and matrix metalloproteinase with thrombospondin motif-5 (ADAMTS-5) and matrix metalloproteinase-13 (MMP-13) gene expression or on sulphated glycosaminoglycans (sGAG) release in human articular cartilage. Changes in the gene expression of ADAMTS-5 and MMP-13 in isolated human articular chondrocytes are shown after 24 hours (a) and 72 hours (b) of stimulation with recombinant Indian hedgehog ( $r-1 / h h)$ compared to controls. Data are mean fold-change $(n=12$ from three separate donors). c sGAG release for untreated controls, r-Ihh, and cyclopamine at 24-hour intervals over the 72-hour culture period, and cumulatively over the 72-hour culture period. Data are mean sGAG release, normalised to the untreated controls from the same donor tissue at the same time point, with standard deviation error bars $(n=6)$. ${ }^{*}$ Statistically significant difference between groups and their respective untreated controls, ${ }^{*} P<0.05,{ }^{* *} P \leq 0.01$, and ${ }^{* * *} P \leq 0.001$ 


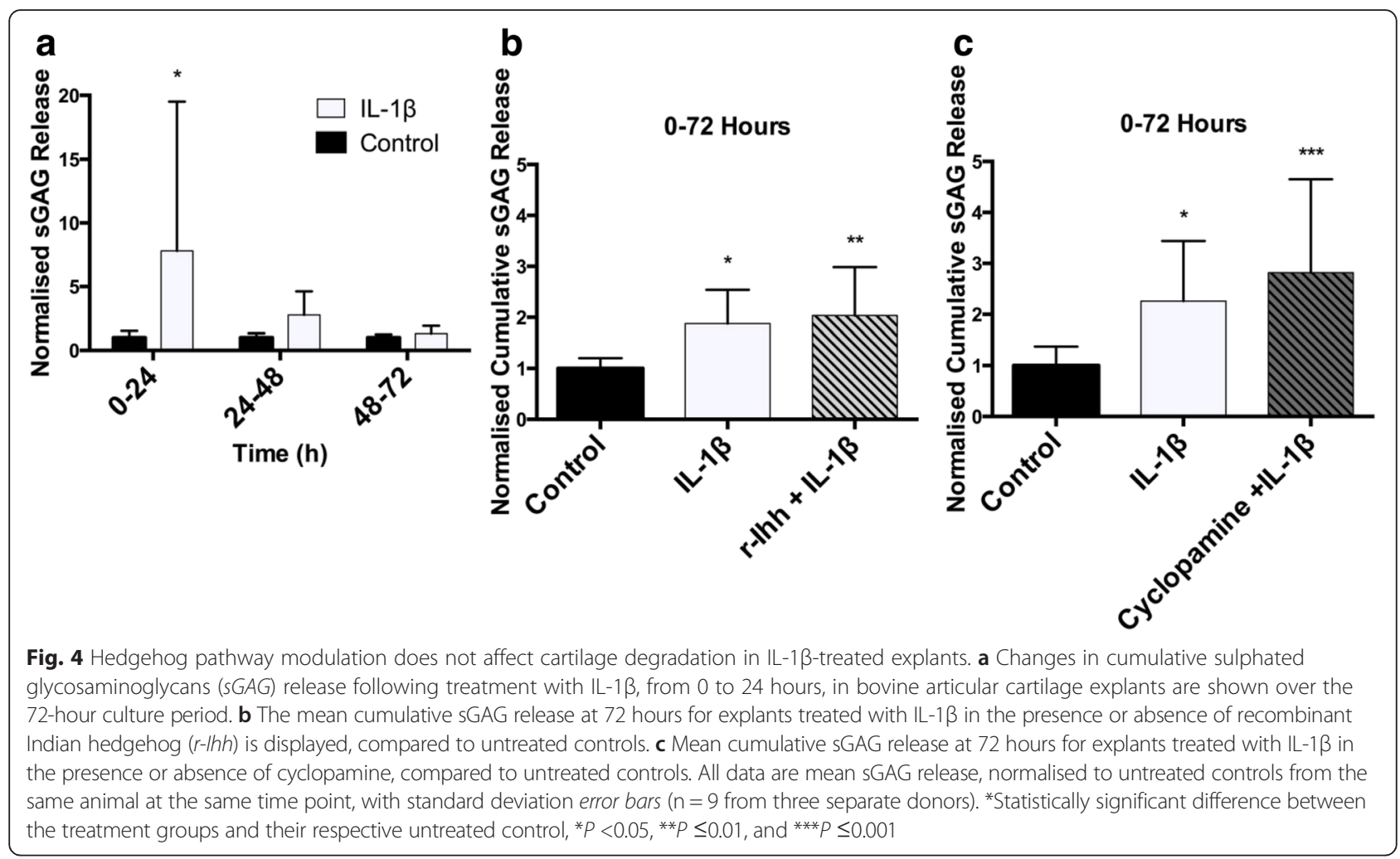

indicate that the presence of IL-1 $\beta$ in this in vitro model of inflammatory arthritis does not modify the catabolic response to hedgehog pathway activation.

\section{IL-1 $\beta$ suppresses hedgehog pathway activation in bovine articular chondrocytes}

Finally, we examined whether IL-1 $\beta$ influenced the activation of hedgehog signalling in response to the addition of r-Ihh (Fig. 5). Treatment of isolated bovine chondrocytes with $10 \mathrm{ng} / \mathrm{mL}$ IL-1 $\beta$ inhibited $\mathrm{r}$-Ihh-induced Gli1 (Fig. 5a) and Ptch1 (Fig. 5b) gene expression. Conversely, we also observed that activation of hedgehog signalling weakly downregulates IL-1 $\beta$-induced ADAMTS-5 expression (Fig. 5c). These results suggest there is molecular crosstalk between the hedgehog and IL-1 $\beta$ signalling pathways in chondrocytes.
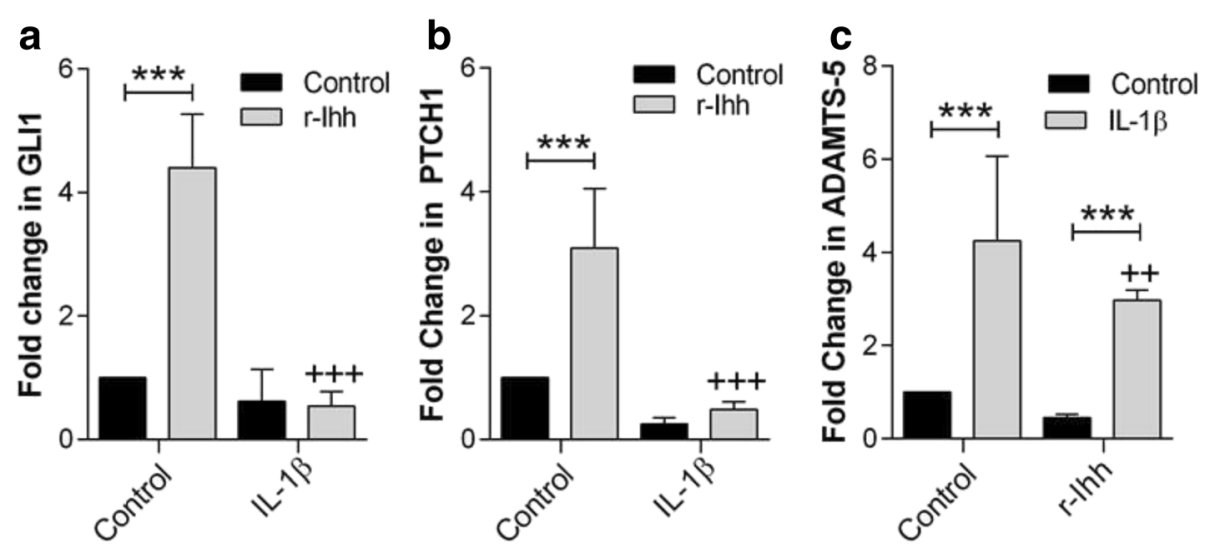

Fig. 5 IL-1 $\beta$ reduces hedgehog pathway activation in response to recombinant Indian hedgehog ( $r-l h h)$. After 24 hours of treatment, in bovine articular chondrocytes, IL-1 $\beta$ prevents upregulation in GLI1 (a) and PTCH1 (b) expression in response to r-Ihh treatment. c Treatment with IL-1 3 similarly repressed $\mathrm{r}$-lhh upregulation of a disintegrin and matrix metalloproteinase with thrombospondin motif (ADAMTS-5) expression but did not completely abolish this effect. *Statistically significant difference between the $r$-lhh-treated samples and the untreated control; ${ }^{+}$statistically significant difference between the $r-\mathrm{lhh} / \mathrm{LL}-1 \beta$-treated samples and the $\mathrm{r}$-lhh-treated samples $\left(\mathrm{n}=9\right.$ from three separate donors), ${ }^{* * *} P \leq 0.001$, ${ }^{++} P \leq 0.01$, and ${ }^{+++} P \leq 0.001$ 


\section{Discussion}

In this study, we examined the effects of hedgehog signalling on ADAMTS-5 and MMP-13 gene expression and cartilage degradation in isolated chondrocytes and cartilage tissue, and in a well-established in vitro explant model of inflammatory arthritis [19]. The efficacy of this IL-1 $\beta$ treatment protocol has been proven in our previous work, which showed significant effects of IL- $1 \beta$-treatment on a number of parameters such as $\mathrm{PGE}_{2}$, $\mathrm{NO}$, matrix degradation and biomechanical changes [19, 27-29]. Our work is the first to illustrate that ligand-activation of chondrocyte hedgehog signalling is unable to stimulate the expression of the pro-catabolic genes ADAMTS-5 and MMP-13 or directly induce cartilage matrix breakdown. We also demonstrated that even in IL- $1 \beta$ treated explants, the activation or inhibition of hedgehog signalling cascade does not yield any significant effects on cartilage matrix degradation.

As these studies are focused on examining ligandinduced hedgehog signalling, the basal levels of hedgehog signalling was not measured in these studies. We have, however, shown the induction of hedgehog signalling via Ihh-induced upregulation of Gli1 and Ptch1 (Fig. 1a and Fig. 5a and b). Despite successful activation of the hedgehog pathway, the expression of ADAMTS-5 and $M M P-13$ was not significantly altered by $r$-Ihh treatment in bovine or human articular chondrocytes. This lack of catabolic response to hedgehog signalling is an apparent contrast to previous studies [26, 28]. These differences, however, were not due to the loss of cellularity, as shown by cell viability analysis, which was approximately $70 \%$ in all groups including controls at the end of the 72-hour treatment period. In previous studies, hedgehog pathway activation was shown to upregulate the expression of these catabolic genes downstream of the transcription factor Runx2 [5]. It is therefore possible that the 24-hour treatment used in the current study was not sufficient to regulate gene expression. However, ADAMTS-5 and MMP-13 expression were not significantly influenced by $\mathrm{r}$-Ihh in human articular chondrocytes following longer treatment for 72 hours either. Hedgehog signalling also failed to stimulate matrix catabolism in bovine cartilage explants. Similar results were obtained for human cartilage explants, which, however, were taken from a single donor and therefore can only be used as validation for bovine and isolated chondrocyte studies. Overall, these data indicate that hedgehog pathway activation by itself does not stimulate cartilage degradation.

Our results may appear to be in contrast to previous studies linking the hedgehog pathway to ADAMTS-5 and $M M P-13$ expression. For example, Lin et al. demonstrated that $\mathrm{r}$-Ihh stimulation in human cartilage in OA increases the expression of both ADAMTS-5 and MMP-13 [26].
Similarly, Zhou et al. demonstrated that surgically induced $\mathrm{OA}$ in Ihh-deleted mice results in the decreased expression of MMP-13 [27]. However, to the best of our knowledge, this is the first study to examine the effect of Ihh on ADAMTS-5 and MMP-13 expression in healthy chondrocytes, as opposed to the OA models used in previous studies where Ihh signalling deregulated the expression of catabolic enyzmes $[4-6,9,15,17,18,30,31]$. Ihh signalling has been described as "context-dependent" such that it inhibits or promotes chondrocyte hypertrophy depending on the levels of parathyroid hormone-related protein present [32]. It is thus possible that Ihh signalling may be influenced by its interactions with the complex biochemical milieu within cartilage in OA. It is possible however that these alterations in hedgehog signalling may have long-term effects that are not measurable in the time course used in these studies. These include potential effects on chondrocyte fate, including an eventual development of chondrocyte hypertrophy and subsequent upregulation in catabolic enzymes and the development of osteoarthritic phenotype.

In healthy cartilage, Runx 2 expression is inhibited directly by HDAC4 and indirectly by transforming growth factor- $\beta$ (TGF- $\beta$ ), via Smads 2 and 3. Therefore two plausible candidates for interaction are histone deacetylase 4. (HDAC4) and TGF- $\beta[17,33]$. The inhibitory effects of these proteins on Runx 2 may prevent Ihhinduced expression of Runx2 in healthy cartilage, which would explain the lack of Ihh-induced ADAMTS-5 and $M M P-13$ upregulation observed in the current study. In $\mathrm{OA}$, the levels of HDAC4 are decreased, relieving its inhibition of Runx2. Similarly in OA, TGF- $\beta$ activates Smads 1, 5, and 8, which promote Runx2 expression $[17,33]$. It is in this context that Ihh may be able to contribute to the upregulation of Runx2 and subsequent ADAMTS-5- and MMP-13-mediated cartilage degradation in the joints in OA.

In this study, we also examined the influence of hedgehog pathway activation in a well-established in vitro bovine explant model of inflammatory arthritis [34]. We have previously shown that IL-1 $\beta$ induces the trafficking of key signalling proteins onto primary cilia with an associated increase in cilia length [27-29]. As hedgehog signalling in chondrocytes also requires ciliary trafficking, we hypothesised that IL-1 $\beta$ synergises the catabolic response to hedgehog ligand through modulation of the primary cilium. This model is based on the proinflammatory cytokine, IL-1 $\beta$, which is upregulated in inflammatory arthritis and causes cartilage degradation $[19,20,35,36]$. Although there are numerous factors that contribute to the development of $\mathrm{OA}$, including pathological increases in other cytokines, injurious mechanical loading, increased wnt signalling etc., it is challenging to incorporate all these factors into a single 
in vitro model. Bovine cartilage explants were treated with $2.5 \mathrm{ng} / \mathrm{mL}$ of IL-1 $\beta$ from $0-24$ hours and then treated with normal chondrocyte media for the remainder of the 72-hour culture period (Fig. 4). While higher than the long-term pathological concentrations of IL-1 $\beta$ measured within the joints in OA [34, 37], the dosing strategy used in the current study aimed to strike a balance between having a sufficiently high IL- $1 \beta$ dose to induce detectable levels of matrix degradation over a short period of time without overshadowing the possible catabolic effects of hedgehog signalling. IL-1 $\beta$ treatment increased cartilage degradation throughout the 72-hour culture period compared to untreated controls; this was not influenced by treatment with r-Ihh. In addition, the hedgehog antagonists, cyclopamine, KAAD-cyclopamine, SANT-1, and GANT61, did not display any significant inhibition of IL-1 $\beta$-induced cartilage damage, despite previous reports suggesting a potential therapeutic role for hedgehog blockade in arthritis [4-6]. Thus, these data show no synergistic interaction between the catabolic effects of hedgehog signalling and IL-1 $\beta$.

Finally, we showed that in IL-1 $\beta$-treated chondrocytes, the upregulation of Gli1 and Ptch1 in response to r-Ihh is greatly reduced. We also showed that IL-1 $\beta$ induces an upregulation in ADAMTS5 expression, which was significantly reduced but not abolished by Ihh-treatment. We suggest that this is due to the increase in primary cilia length with IL-1 $\beta$ treatment [28] as our recent studies show an inverse relationship between cilia length and hedgehog signalling $[9,38]$. The mechanism for reduced hedgehog signalling may be due to reduced intraflagellar transport $[28,39]$ and changes in ciliary trafficking $[29,40]$.

\section{Conclusion}

Previous studies report that an aberrant upregulation of hedgehog signalling occurs in OA, leading to cartilage degradation [4-6]. However, this study showed for the first time that in healthy cartilage in vitro, hedgehog pathway activation by itself does not cause cartilage degradation. We therefore examined whether the presence of inflammatory cytokines stimulated hedgehog-induced catabolism. However, even in the presence of IL-1 $\beta$, neither hedgehog agonists nor antagonists had any effect on matrix degradation. Thus, we suggest that the reported role of hedgehog signalling in matrix catabolism in OA must be due to a complex interaction with other pathological factors or more long-term effects on chondrocyte fate. Importantly, we demonstrated that IL-1 $\beta$ suppresses hedgehog pathway activation by $\mathrm{r}$-Ihh therefore any possible effects of hedgehog signalling in OA are likely to be downregulated by the presence of inflammatory cytokines. We conclude that chondrocyte hedgehog signalling is not catabolic in otherwise healthy cartilage and is in fact downregulated by inflammatory cytokines, such that any role for hedgehog is extremely context-dependent.

\section{Additional file}

\begin{abstract}
Additional file 1: Figure S1. Hedgehog pathway activation over 6 days does not influence cartilage degradation. Changes in sulphated glycosaminoglycans ( $s G A G$ ) release following treatment with IL-1 $\beta$ or recombinant Indian hedgehog $(r-1 / h)$, in bovine articular cartilage explants, over a 6-day culture period. Although IL-1 $\beta$ increased sGAG release into the media over the 6-day culture period, there was no upregulation in SGAG in response to $r$-lhh treatment ( $n=6$ from three separate donors). Figure S2. Treatment with $r$-lhh or cyclopamine had no effect on chondrocyte viability. a Representative fluorescence microscopy image showing live cells (calcein AM/green) and dead cells (ethidium homodimer/red) within a cartilage explant. Scale bar represents $50 \mu \mathrm{m}$. Arrows indicate the articular surface. b Percentage cell viability. Values represent mean with error bars showing standard deviations ( $n=9$ from three separate donors). Figure S3. Hedgehog pathway antagonists KAAD-cyclopamine, SANT-1, and GANT61 do not inhibit cartilage degradation in IL-1 $1 \beta$-treated explants. Following treatment with IL-1 $\beta$ for 24 hours, bovine cartilage explants exhibit an increase in cumulative sGAG release after 72 hours compared to untreated controls, however, the hedgehog antagonists KAAD-cyclopamine, SANT-1, and GANT61 did not significantly modulate the effects of IL-1 $\beta$. All data are mean SGAG release, normalised to untreated controls from the same animal at the same time point ( $n=9$ from three separate donors). *Statistically significant difference, ${ }^{*} P<0.05,{ }^{* *} P \leq 0.01$, and ${ }^{* * *} P \leq 0.001$. (DOCX $1215 \mathrm{~kb}$ )
\end{abstract}

\begin{abstract}
Abbreviations
ACAN: aggrecan gene; ADAMTS: a disintegrin and matrix metalloproteinase with thrombospondin motif; BSA: bovine serum albumin; COL2A1: type II collagen gene; COX-2: prostaglandin $E_{2}$ producing enzyme; DMEM: Dulbecco's modified Eagle's medium; DMMB: dimethymethylene blue; FBS: foetal bovine serum; HDAC4: histone deacetylase 4; Ihh: Indian hedgehog; IL-1ß: interleukin-1 $\beta$; iNOS: nitric oxide producing enzyme; MMP: matrix metalloproteinase; NO: nitric oxide; OA: osteoarthritis; PBS: phosphate-buffered saline; $\mathrm{PGE}_{2}$ : prostaglandin $\mathrm{E}_{2}$; Ptch1: patched 1; r-lhh: recombinant Ihh; Runx2: runt-related transcription factor 2; sGAG: sulphated glycosaminoglycans; Smo: Smoothened; TGF $\beta$ : transforming growth factor $\beta$.
\end{abstract}

\section{Competing interests}

The authors declare that they have no competing interests.

\section{Authors' contributions}

$\mathrm{CT}$ conducted isolated chondrocyte as well as the human explant experiments and helped in drafting the manuscript. RP conducted the bovine explant experiments and drafted the manuscript. TK assisted with the bovine explant experiments and provided substantial editing of the manuscript. AW, JC, CH, and MK provided the conceptual framework for these studies and editing for the manuscript. All authors were involved in data analysis and read and approved the final manuscript.

\section{Acknowledgements}

AW and TK were funded on an Arthritis Research UK (3514) project grant. $\mathrm{CT}$ was funded on an $\mathrm{AO}$ Foundation (S-12-15 K) project grant and is now on an MRC (MR/L002876/1) project grant. RP conducted the work as part of an intercalated BSc in Biomedical Engineering \& Clinical Materials.

\section{Author details}

${ }^{1}$ Institute of Bioengineering and School of Engineering and Material Sciences, Queen Mary University of London, Mile End Road, London E1 4NS, UK. ${ }^{2}$ Center for Endocrinology, William Harvey Research Institute, School of Medicine and Dentistry, Queen Mary University of London, London, UK. ${ }^{3}$ Department of Biomedical Engineering, Columbia University, New York, NY, USA. ${ }^{4}$ Kennedy Institute of Rheumatology, University of Oxford, Oxford, UK.

Received: 7 September 2015 Accepted: 8 December 2015

Published online: 24 December 2015 


\section{References}

1. Sandell LJ, Aigner T. Articular cartilage and changes in arthritis. An introduction: cell biology of osteoarthritis. Arthritis Res. 2001;3(2):107-13.

2. Sellam J, Berenbaum F. Is osteoarthritis a metabolic disease? Joint Bone Spine. 2013;80(6):568-73

3. Berenbaum F. Osteoarthritis as an inflammatory disease (osteoarthritis is not osteoarthrosis!). Osteoarthritis Cartilage. 2013;21(1):16-21.

4. Wei F, Zhou J, Wei X, Zhang J, Fleming BC, Terek R, et al. Activation of Indian hedgehog promotes chondrocyte hypertrophy and upregulation of MMP-13 in human osteoarthritic cartilage. Osteoarthritis Cartilage. 2012;20(7):755-63.

5. Lin AC, Seeto BL, Bartoszko JM, Khoury MA, Whetstone $H$, Ho L, et al. Modulating hedgehog signaling can attenuate the severity of osteoarthritis. Nat Med. 2009;15(12):1421-5.

6. Zhou J, Chen Q, Lanske B, Fleming BC, Terek R, Wei X, et al. Disrupting the Indian hedgehog signaling pathway in vivo attenuates surgically induced osteoarthritis progression in Col2a1-CreERT2. Ihhfl/fl mice Arthritis Res Ther. 2014;16(1):R11.

7. Corbit KC, Aanstad P, Singla V, Norman AR, Stainier DY, Reiter JF. Vertebrate Smoothened functions at the primary cilium. Nature. 2005;437(7061):1018-21.

8. Rohatgi R, Milenkovic L, Scott MP. Patched1 regulates hedgehog signaling at the primary cilium. Science. 2007;317(5836):372-6.

9. Thompson $\mathrm{CL}$, Chapple JP, Knight MM. Primary cilia disassembly down-regulates mechanosensitive hedgehog signalling: a feedback mechanism controlling ADAMTS-5 expression in chondrocytes. Osteoarthritis Cartilage. 2014;22(3):490-8.

10. Huangfu D, Liu A, Rakeman AS, Murcia NS, Niswander L, Anderson KV. Hedgehog signalling in the mouse requires intraflagellar transport proteins. Nature. 2003:426(6962):83-7.

11. Buttitta L, Mo R, Hui CC, Fan CM. Interplays of Gli2 and Gli3 and their requirement in mediating Shh-dependent sclerotome induction. Development. 2003;130(25):6233-43.

12. St-Jacques $B$, Hammerschmidt M, McMahon AP. Indian hedgehog signaling regulates proliferation and differentiation of chondrocytes and is essential for bone formation. Genes Dev. 1999;13(16):2072-86.

13. Vortkamp A, Lee K, Lanske B, Segre GV, Kronenberg HM, Tabin CJ. Regulation of rate of cartilage differentiation by Indian hedgehog and PTH-related protein. Science. 1996;273(5275):613-22.

14. van der Kraan PM, van den Berg WB. Chondrocyte hypertrophy and osteoarthritis: role in initiation and progression of cartilage degeneration? Osteoarthritis Cartilage. 2012;20(3):223-32.

15. Zhang C, Wei X, Chen C, Cao K, Li Y, Jiao Q, et al. Indian hedgehog in synovial fluid is a novel marker for early cartilage lesions in human knee joint. Int J Mol Sci. 2014;15(5):7250-65.

16. Shimoyama A, Wada M, Ikeda F, Hata K, Matsubara T, Nifuji A, et al. Ihh/Gli2 signaling promotes osteoblast differentiation by regulating Runx2 expression and function. Mol Biol Cell. 2007;18(7):2411-8.

17. Cao K, Wei L, Zhang Z, Guo L, Zhang C, Li Y, et al. Decreased histone deacetylase 4 is associated with human osteoarthritis cartilage degeneration by releasing histone deacetylase 4 inhibition of runt-related transcription factor-2 and increasing osteoarthritis-related genes: a novel mechanism of human osteoarthritis cartilage degeneration. Arthritis Res Ther. 2014;16(6):491.

18. Tchetina EV, Squires G, Poole AR. Increased type II collagen degradation and very early focal cartilage degeneration is associated with upregulation of chondrocyte differentiation related genes in early human articular cartilage lesions. J Rheumatol. 2005:32(5):876-86.

19. Wann AK, Mistry J, Blain EJ, Michael-Titus AT, Knight MM. Eicosapentaenoic acid and docosahexaenoic acid reduce interleukin-1beta-mediated cartilage degradation. Arthritis Res Ther. 2010;12(6):R207.

20. Wang SN, Xie GP, Qin CH, Chen YR, Zhang KR, Li X, et al. Aucubin prevents interleukin-1 beta induced inflammation and cartilage matrix degradation via inhibition of NF-kappaB signaling pathway in rat articular chondrocytes. Int Immunopharmacol. 2015;24(2):408-15

21. Chen JK, Taipale J, Cooper MK, Beachy PA. Inhibition of Hedgehog signaling by direct binding of cyclopamine to Smoothened. Genes Dev. 2002;16(21):2743-8.

22. Li R, Cai L, Ding J, Hu CM, Wu TN, Hu XY. Inhibition of hedgehog signal pathway by cyclopamine attenuates inflammation and articular cartilage damage in rats with adjuvant-induced arthritis. J Pharm Pharmacol. 2015;67(7):963-71.

23. Rohatgi R, Milenkovic L, Corcoran RB, Scott MP. Hedgehog signal transduction by Smoothened: pharmacologic evidence for a 2-step activation process. Proc Natl Acad Sci U S A. 2009;106(9):3196-201.

24. Watkins DN, Berman DM, Burkholder SG, Wang B, Beachy PA, Baylin SB. Hedgehog signalling within airway epithelial progenitors and in small-cell lung cancer. Nature. 2003;422(6929):313-7.
25. Chowdhury TT, Bader DL, Lee DA. Dynamic compression inhibits the synthesis of nitric oxide and PGE(2) by IL-1beta-stimulated chondrocytes cultured in agarose constructs. Biochem Biophys Res Commun. 2001;285(5):1168-74.

26. Farndale RW, Sayers CA, Barrett AJ. A direct spectrophotometric microassay for sulfated glycosaminoglycans in cartilage cultures. Connect Tissue Res. 1982;9(4):247-8.

27. Wann AK, Chapple JP, Knight MM. The primary cilium influences interleukin1 beta-induced NFkappaB signalling by regulating IKK activity. Cell Signal. 2014;26(8):1735-42.

28. Wann AK, Knight MM. Primary cilia elongation in response to interleukin-1 mediates the inflammatory response. Cell Mol Life Sci. 2012:69(17):2967-77.

29. Wann AK, Thompson CL, Chapple JP, Knight MM. Interleukin-1beta sequesters hypoxia inducible factor 2alpha to the primary cilium. Cilia. 2013;2(1):17.

30. Alman BA. The role of hedgehog signalling in skeletal health and disease. Nat Rev Rheumatol. 2015;11(9):552-60.

31. Zhou J, Wei X, Wei L. Indian Hedgehog, a critical modulator in osteoarthritis, could be a potential therapeutic target for attenuating cartilage degeneration disease. Connect Tissue Res. 2014;55(4):257-61.

32. Mak KK, Kronenberg HM, Chuang PT, Mackem S, Yang Y. Indian hedgehog signals independently of PTHrP to promote chondrocyte hypertrophy. Development. 2008;135(11):1947-56.

33. van der Kraan PM, Blaney Davidson EN, Blom A, van den Berg WB. TGF-beta signaling in chondrocyte terminal differentiation and osteoarthritis: modulation and integration of signaling pathways through receptor-Smads. Osteoarthritis Cartilage. 2009;17(12):1539-45.

34. Kokebie R, Aggarwal R, Lidder S, Hakimiyan AA, Rueger DC, Block JA, et al. The role of synovial fluid markers of catabolism and anabolism in osteoarthritis, rheumatoid arthritis and asymptomatic organ donors. Arthritis Res Ther. 2011;13(2):R50

35. Qin J, Shang L, Ping AS, Li J, Li XJ, Yu H, et al. Response to TNF/TNFR signal transduction pathway-mediated anti-apoptosis and anti-inflammatory effects of sodium ferulate on IL-1beta-induced rat osteoarthritis chondrocytes in vitro'- authors' reply. Arthritis Res Ther. 2013;15(3):409.

36. Stabellini G, De Mattei M, Calastrini C, Gagliano N, Moscheni C, Pasello M, et al. Effects of interleukin-1beta on chondroblast viability and extracellular matrix changes in bovine articular cartilage explants. Biomed Pharmacother 2003:57(7):314-9.

37. McNulty AL, Rothfusz NE, Leddy HA, Guilak F. Synovial fluid concentrations and relative potency of interleukin-1 alpha and beta in cartilage and meniscus degradation. J Orthop Res. 2013;31(7):1039-45.

38. Prodromou NV, Thompson CL, Osborn DP, Cogger KF, Ashworth R, Knight MM, et al. Heat shock induces rapid resorption of primary cilia. J Cell Sci. 2012;125(Pt 18):4297-305.

39. Wang Y, Zhou Z, Walsh CT, McMahon AP. Selective translocation of intracellular Smoothened to the primary cilium in response to Hedgehog pathway modulation. Proc Natl Acad Sci U S A. 2009;106(8):2623-8.

40. Larkins CE, Aviles GD, East MP, Kahn RA, Caspary T. Arl13b regulates ciliogenesis and the dynamic localization of Shh signaling proteins. Mol Biol Cell. 2011;22(23):4694-703.

41. Stanton BZ, Peng LF. Small-molecule modulators of the Sonic Hedgehog signaling pathway. Mol Biosyst. 2010;6(1):44-54.

42. Kim J, Hsia EY, Kim J, Sever N, Beachy PA, Zheng X. Simultaneous measurement of smoothened entry into and exit from the primary cilium. PLoS One. 2014:9(8):e104070.

43. Wang C, Wu H, Evron T, Vardy E, Han GW, Huang XP, et al. Structural basis for Smoothened receptor modulation and chemoresistance to anticancer drugs. Nat Commun. 2014:5:4355.

44. Chen JK, Taipale J, Young KE, Maiti T, Beachy PA. Small molecule modulation of Smoothened activity. Proc Natl Acad Sci U S A. 2002;99(22):14071-6.

45. Lauth M, Bergstrom A, Shimokawa T, Toftgard R. Inhibition of GLI-mediated transcription and tumor cell growth by small-molecule antagonists. Proc Natl Acad Sci U S A. 2007;104(20):8455-60.

46. Mazumdar T, Devecchio J, Agyeman A, Shi T, Houghton JA. Blocking Hedgehog survival signaling at the level of the GLI genes induces DNA damage and extensive cell death in human colon carcinoma cells. Cancer Res. 2011:71(17):5904-14. 